\title{
Prevalence of malnutrition and associated factors among under-five children in Ethiopia: evidence from the 2016 Ethiopia Demographic and Health Survey
}

\author{
Abay Kassa Tekile* ${ }^{*}$, Ashenafi Abate Woya and Garoma Wakjira Basha
}

\begin{abstract}
Objective: The aim of this study was to assess the risk factors for malnutrition among children aged 0-59 months in Ethiopia. The analyzed data were obtained from the 2016 EDHS and 9495 under-5 years' children were considered in this analysis. The data was extracted, edited and analyzed by using SPSS Version 23.0. Both bivariate and multivariable binary logistic regression model was used to identify the determinants of children malnutrition.

Results: The prevalence of stunting, wasting, and underweight were $38.3 \%, 10.1 \%$, and $23.3 \%$, respectively. About $19.47 \%$ of children were both stunted and underweighted, and only $3.87 \%$ of children had all the three conditions. Among the factors that considered in this study, age of a child, residence region, mothers' education level, mothers' BMI, household wealth index, sex of a child, family size, water and toilet facility were significantly associated with malnutrition in Ethiopia. The authors concluded that malnutrition among under-five children was one of the public health problems in Ethiopia. Therefore, the influence of these factors should be considered to develop strategies for reducing malnutrition in Ethiopia.
\end{abstract}

Keywords: Under-five children, Underweight, Wasting, Stunting, Ethiopia

\section{Introduction}

Malnutrition among under- 5 year children is a common public health problem and it is one of the main reasons for the death of children in developing countries [1]. As of the World Health Organization report, about 35\% of under-five children's death is associated with malnutrition in the world [2]. There are 165 million stunted, 99 million under-weighted, and 51 million wasted children globally [3].

The prevalence of stunting has decreased from $58 \%$ in 2000 to $44 \%$ in 2011 in Ethiopia. The prevalence of wasting is changed from $12 \%$ in 2000 to $10 \%$ in 2011 . The prevalence of underweight has consistently decreased from $41 \%$ in 2000 to $29 \%$ in 2011 [4]. In Tanzania, a high

\footnotetext{
*Correspondence: abaystat@gmail.com
}

Statistics Department, Science College, Bahir Dar University, P.O. Box 79, Bahir Dar, Ethiopia prevalence of underweight (46.0\%), stunting (41.9\%) and wasting (24.7\%) are observed in 2017. In addition, 33\% of children are both stunted and underweight, $21 \%$ of children are underweight and wasted, and $12 \%$ of children are stunted and wasted [5]. In Ethiopia, more than onethird of a child deaths are associated with malnutrition [6]. Moreover, the proportion of malnutrition is higher among anemic children compared to those of non-anemic [7].

Different researchers conducted a study on malnutrition among under-five children in different parts of the country. These studies were mainly focused on the prevalence and determinants of malnutrition among under-five children, but they gave little attention for exploring the relationship between under-five malnutrition and child anemia. So, the main aim of this study was to explore the major factors of malnutrition and its association with anemia by using updated data from the 2016 EDHS. 


\section{Main text}

\section{Study design and sampling}

The 2016 Ethiopia Demographic and Health Survey data was used for this study. The 2016 EDHS used a twostage stratified sampling design to select households. In the first stage, there were 645 enumeration areas (202 in urban and 443 in rural areas) based on the 2007 Ethiopia Population and Housing Census (PHC). A total of 18,008 households were considered, of which 16,650 (98\% of response rate) households were eligible. The women were interviewed by distributing questio-ners and information on their birth history and 9495 under-five children were considered for this study [8].

\section{Measurements}

The dependent variable for this study was the malnutrition status of under-5 year children (stunting, underweight and wasting). Children whose height-for-age Z-score is below minus two standard deviations ( $-2 \mathrm{SD})$ from the median of the reference population are considered as stunted. If the weight-for-age Z-score is below minus two standard deviations $(-2 \mathrm{SD})$ from the median of the reference population then the child is underweight. Children whose weight for height Z-score is below minus two standard deviations $(-2 \mathrm{SD})$ from the median of the reference population are considered as wasted [8]. Sociodemographic, socio-economic and health-related variables were considered as independent variables in this study.

\section{Statistical data analysis}

The data were extracted, edited, and analyzed by using SPSS version 23 for Windows. Then a weighted analysis was conducted using the same sampling weight given for each region in Ethiopia DHS to compensate for the unequal probability of selection between the strata $[8,12]$. Bivariate logistic regression was performed and variable with P-value less than 0.25 were transported into multivariable binary logistic regression analysis to identify the determinant of malnutrition of under-five children. Finally, variables with P-values $<0.05$ in the multivariable logistic regression model were taken as statistically significant.

\section{Results}

Samples of 9495 under-five children were considered in this research. The weighted prevalence of stunting, underweight, and wasting were $38.3 \%, 23.3 \%$, and $10.1 \%$, respectively. About $66 \%$ of interviewed mothers had no education and only $2 \%$ of them attended higher education. About $44 \%$ of children were found between 0 to 24 months and more than half (51.1\%) were males. Only $11 \%$ of the respondents were from urban areas and $32 \%$ were in the rich wealth index. Around 20\% of children's mother were underweighted (having body mass index less than 18.5) (Additional file 1).

\section{Determinants of stunting}

Among the factors that considered in this study, child's age, residence region, mothers' education level, wealth index, child sex, toilet facility, size of a child, mothers' BMI and number of children per household were associated with stunting. Compared to children of 0-24 months, the odds of stunting among children in the age group of 25-47 months were 2.645 times higher. The child in the age group of 48-59 months was 1.763 times higher. Compared with children in Tigray region, the risk of being stunted was decreased by $32 \%, 33 \%$, and $60 \%$, among children living in Afar, Oromia, and Somali regions, respectively. The risk of being stunted among children whose mothers attended primary education was 0.87 times less compared to children whose mother did not attend education. The risk of being stunted among children whose mothers attended secondary and higher education were 0.606 and 0.453 times less compared to children whose mother did not attend education respectively. Compared to male children, the probability of being stunted among female children was decreased by $16 \%$. Compared to children living in households with poor economic status, the odds of being stunted among children living in households with medium and rich economic status were decreased by $20 \%$ and $31 \%$, respectively.

Children born with small size were 1.509 times more likely to be stunted than children born larger $(\mathrm{AOR}=1.509 ; 95 \% \mathrm{CL} 1.332-1.709)$ and children who had born with medium size were 1.189 times more likely to be stunted than children born larger $(\mathrm{AOR}=1.062$; 95\% CL 1.062-1.331). Children born to underweight mothers $(\mathrm{BMI}<18.5)$ were 2.163 (AOR: 2.163 , 95\% CI $1.750,2.673)$ times more likely to be stunted compared to those born to overweight mothers (Table 1).

\section{Determinants of under-weight}

Age of child, sex of a child, mothers' education level, mothers' BMI, region, household wealth index, water facility, toilet facility, size of child and a number children were associated with under-weight $(\mathrm{P}<0.05)$. The risk of being underweighted was 1.748, 1.837 times more likely among children that were aged $24-47$, and 48-59 months than those aged 0-24 months. Compared to Tigray region, the odds of being under-weighted was $0.741,0.664$ and 0.393 times lower among children from Oromia, Gambella and Addis Ababa respectively.

The risk of being underweight for children whose mother attend primary, secondary and higher education 
Table 1 Determinants associated to stunting (EDHS, 2016)

\begin{tabular}{|c|c|c|c|c|}
\hline Variables & P-value & COR $(95 \% \mathrm{Cl})$ & P-value & AOR $(95 \% \mathrm{Cl})$ \\
\hline \multicolumn{5}{|l|}{ Age of child in months } \\
\hline $0-24$ (ref) & $<0.0001$ & 1 & $<0.0001$ & 1 \\
\hline $25-47$ & $<0.0001$ & $2.565(2.320,2.836)$ & $<0.0001$ & $2.645(2.384,2.935)$ \\
\hline $48-59$ & $<0.0001$ & $1.750(1.551,2.973)$ & $<0.0001$ & $1.763(1.557,1.997)$ \\
\hline \multicolumn{5}{|l|}{ Region of residence } \\
\hline Tigray (ref) & $<0.0001$ & 1 & $<0.0001$ & 1 \\
\hline Afar & 0.101 & $1.078(0.688,1.689)$ & 0.003 & $0.683(0.530,0.880)$ \\
\hline Amhara & 0.003 & $1.398(1.165,1.678)$ & 0.113 & $1.173(0.963,1.428$ \\
\hline Oromia & 0.055 & $0.890(0.751,1.055)$ & $<0.0001$ & $0.670(0.538,0.834$ \\
\hline Somali & $<0.0001$ & $0.581(0.442, .764)$ & $<0.0001$ & $0.401(0.312,0.515$ \\
\hline Benishangul-gumuz & 0.097 & $1.161(0.758,1.780)$ & 0.429 & $0.911(0.723,1.148)$ \\
\hline SNNPR & 0.468 & $0.999(0.832,1.199)$ & 0.062 & $0.800(0.633,1.011$ \\
\hline Gambela & $<0.0001$ & $0.480(0.177,1.306)$ & $<0.0001$ & $0.467(0.350,0.625$ \\
\hline Harar & 0.004 & $0.739(0.282,1.933)$ & 0.009 & $0.679(0.509,0.908$ \\
\hline Addis Ababa & $<0.0001$ & $0.271(0.179, .409)$ & $<0.0001$ & $0.476(0.338,0.671$ \\
\hline Dire dawa & 0.945 & $1.108(0.562,2.187)$ & 0.413 & $0.886(0.664,1.183$ \\
\hline \multicolumn{5}{|l|}{ Mother's education level } \\
\hline No education (ref) & $<0.0001$ & 1 & $<0.0001$ & 1 \\
\hline Primary & $<0.0001$ & $0.758(0.685, .840)$ & 0.016 & $0.870(0.777,0.974$ \\
\hline Secondary & $<0.0001$ & $0.401(0.328,0.491)$ & $<0.0001$ & $0.606(0.483,0.761$ \\
\hline Higher & 0.0001 & $0.247(0.179, .340)$ & $<0.0001$ & $0.453(0.319,0.643$ \\
\hline \multicolumn{5}{|l|}{ Mother's BMI } \\
\hline Overweight (ref) & $<0.0001$ & 1 & $<0.001$ & 1 \\
\hline Thin for height & $<0.001$ & $2.167(1.759,2.668$ & $<0.001$ & $2.163(1.750,2.673$ \\
\hline Normal & $<0.001$ & $1.993(1.643,2.418$ & $<0.001$ & $1.993(1.638,2.426$ \\
\hline \multicolumn{5}{|l|}{ Household wealth index } \\
\hline Poor (ref) & $<0.001$ & 1 & $<0.001$ & 1 \\
\hline Medium & $<0.0001$ & $0.772(0.693,0.860)$ & 0.002 & $0.801(0.697,0.921)$ \\
\hline Rich & $<0.001$ & $0.555(0.504 .0 .612)$ & $<0.001$ & $0.688(0.606,0.781)$ \\
\hline \multicolumn{5}{|l|}{ Sex of a child } \\
\hline Female & $<0.001$ & $0.862(0.790,0.941)$ & $<0.001$ & $0.839(0.765,0.920)$ \\
\hline \multicolumn{5}{|l|}{ Size of a child at birth } \\
\hline Large (ref) & $<0.001$ & 1 & $<0.001$ & 1 \\
\hline Medium & 0.064 & $1.098(0.995,1.212)$ & 0.003 & $1.189(1.062,1.331$ \\
\hline Small & $<0.001$ & $1.475(1.323,1.64)$ & $<0.001$ & $1.509(1.332,1.709$ \\
\hline \multicolumn{5}{|l|}{ Toilet facility } \\
\hline Have facilities & $<0.001$ & $0.520(0.442,0.61)$ & $<0.001$ & $0.758(0.642,0.896$ \\
\hline \multicolumn{5}{|l|}{ Number of children } \\
\hline No (ref) & 0.003 & 1 & $<0.001$ & 1 \\
\hline $1-2$ & 0.523 & $0.860(0.543,1.365$ & 0.013 & $0.475(0.264,0.853$ \\
\hline $3-4$ & 0.134 & $0.698(0.436,1.117$ & $<0.001$ & $0.342(0.188,0.620$ \\
\hline$>4$ & 0.596 & $0.826(0.407,1.677$ & 0.019 & $0.363(0.155,0.849$ \\
\hline
\end{tabular}

ref: reference category; COR: crude odds ratio; AOR: adjusted odds ratio; Cl: confidence interval

were $0.771,0.645$, and 0.551 times lower than children whose mothers who did not attend formal education. Children from a household with middle and rich economic status were 0.794 and 0.565 times less likely to be under-weighted compared to children living in a household with poor household economic status.

Female children were 0.856 times less likely to be under-weighted as compared to male children. Children 
who were born with small size were 1.898 times more likely to be under-weighted than children born larger $(\mathrm{AOR}=1.898 ; 95 \%$ CL 1.653-2.180) and children who had born with medium size were 1.324 times more likely to be under-weighted than children born larger $(\mathrm{AOR}=1.324 ; 95 \% \mathrm{CL} 1.164-1.507)$. Children born to underweight mothers $(\mathrm{BMI}<18.5)$ were 3.162 (AOR: 3.162, 95\% CI: $2.410,4.148$ ) times more likely to be underweight compared to Children born to overweight mother (Table 2).

\section{Determinants of wasting}

Results of multivariable binary logistic regression model showed that the age of a child, sex of a child, mothers' education level, household wealth index, a region of residence, water facility, and family size were significantly

Table 2 Significant determinants related to underweight (EDHS, 2016)

\begin{tabular}{|c|c|c|c|c|}
\hline Variable & P-value & COR $(95 \% \mathrm{Cl})$ & P-value & AOR $(95 \% \mathrm{Cl})$ \\
\hline \multicolumn{5}{|l|}{ Age of child in months } \\
\hline $0-24$ & $<0.001$ & 1 & $<0.001$ & 1 \\
\hline $24-47$ & $<0.001$ & $1.688(1.511,1.886)$ & $<0.001$ & $1.748(1.54,1.965)$ \\
\hline $48-59$ & $<0.001$ & $1.706(1.497,1.943)$ & $<0.001$ & $1.837(1.596,2.113)$ \\
\hline \multicolumn{5}{|l|}{ Region of residence } \\
\hline Tigray (ref) & $<0.001$ & 1 & $<0.001$ & 1 \\
\hline Afar & 0.010 & $1.855(1.159,2.969)$ & 0.324 & $1.152(0.870,1.524)$ \\
\hline Amhara & 0.003 & $1.381(1.119,1.703)$ & 0.218 & $1.149(0.921,1.433)$ \\
\hline Oromia & 0.808 & $0.976(0.800,1.190)$ & 0.020 & $0.741(0.575,0.954)$ \\
\hline Somali & 0.047 & $1.338(1.004,1.783)$ & 0.481 & $0.905(0.686,1.194)$ \\
\hline Benishangul-gumuz & 0.011 & $1.796(1.143,2.825)$ & 0.012 & $1.389(1.076,1.793)$ \\
\hline SNNPR & 0.402 & $0.912(0.737,1.130)$ & 0.010 & $0.698(0.530,0.918)$ \\
\hline Gambela & 0.590 & $0.737(0.242,2.240)$ & 0.015 & $0.664(0.477,0.925)$ \\
\hline Harar & 0.826 & $0.883(0.290,2.686)$ & 0.552 & $0.904(0.647,1.262)$ \\
\hline Addis Ababa & $<0.001$ & $0.190(0.101,0.358)$ & $<0.001$ & $0.393(0.231,0.667)$ \\
\hline Dire dawa & 0.525 & $1.278(0.600,2.720)$ & 0.647 & $1.080(0.778,1.500)$ \\
\hline \multicolumn{5}{|l|}{ Mothers' education level } \\
\hline No education (ref) & $<0.001$ & 1 & $<0.001$ & 1 \\
\hline Primary & $<0.001$ & $0.579(0.514,0.652)$ & $<0.001$ & $0.771(0.673,0.884)$ \\
\hline Secondary & $<0.001$ & $0.325(0.253,0.419)$ & $<0.003$ & $0.645(0.486,0.858)$ \\
\hline Higher & $<0.001$ & $0.188(0.122,0.288)$ & $<0.012$ & $0.551(0.347,0.876)$ \\
\hline \multicolumn{5}{|l|}{ Mothers'BMI } \\
\hline Overweight (ref) & 0.001 & 1 & $<0.001$ & 1 \\
\hline Thin for height & 0.001 & $3.278(2.504,4.291)$ & $<0.001$ & $3.162(2.410,4.148)$ \\
\hline Normal & 0.001 & $2.309(1.786,2.985)$ & $<0.001$ & $2.245(1.733,2.908)$ \\
\hline \multicolumn{5}{|l|}{ Household wealth index } \\
\hline Poor (ref) & $<0.001$ & 1 & $<0.001$ & 1 \\
\hline Medium & $<0.001$ & $0.735(0.650,0.832)$ & 0.003 & $0.794(0.681,0.927)$ \\
\hline Rich & $<0.001$ & $0.473(0.421,0.531)$ & $<0.001$ & $0.565(0.487,0.654)$ \\
\hline \multicolumn{5}{|l|}{ Sex of a child } \\
\hline Female & 0.013 & $0.885(0.803,0.975)$ & 0.003 & $0.856(0.773,0.948)$ \\
\hline Size of a child at birth & $<0.001$ & 1 & & \\
\hline Large (ref) & $<0.001$ & $1.286(1.141,1.449)$ & $<0.001$ & 1 \\
\hline Medium & $<0.001$ & $2.085(1.839,2.364)$ & $<0.001$ & $1.324(1.164,1.507)$ \\
\hline Small & & & $<0.001$ & $1.898(1.653,2.180)$ \\
\hline Water facility & 0.034 & $1.117(1.008,1.238)$ & & \\
\hline Safe & $<0.001$ & $0.562(0.462,0.484)$ & 0.012 & $1.151(1.031,1.284)$ \\
\hline \multicolumn{5}{|l|}{ Toilet facility } \\
\hline Have facility & $<0.001$ & $0.403(0.342,0.474)$ & 0.005 & $0.757(0.623,0.920)$ \\
\hline
\end{tabular}


associated with wasting. Children of the rich household were less likely to be wasted compared to children living in a household with poor household economic status. The risk of being wasted was 0.52 and 0.607 times lower among children of 25-47 and 48-59 months than those 0-24 months. Compared to children from the Tigray region, the odds of being wasted of children from Somali region was 1.671 times higher. The odds of being wasted in SNNPR and Addis Ababa were 0.365 and 0.338 times lower compared to Tigray region respectively. The odds of being wasted was 0.778 times lower among female children than male children (AOR $=0.778,95 \%$ CI 0.681 , 0.889 ). The odds of being wasted was 1.223 times higher among children who lived in household members of 6-10 than children who had lived in household members of $1-5(\mathrm{AOR}=1.223,95 \%$ CI $1.066,1.403)$ (Additional file 2).

\section{Associations between children's anemia and malnutrition}

This study showed that among stunted, underweighted, and wasted children, $61 \%, 64.3 \%$, and $68.2 \%$ were anemic respectively. Moreover, the percentages of stunting, wasting, and underweighting were higher among anemic children as compared to no-anemic children. Stunted children were 1.222 times more likely to be anemic compared to those of not stunted (AOR: 1.222, 95\% CI 1.101, 1.356). Underweighted children were 1.222 times more likely to be anemic compared to those of not underweight (AOR: 1.222, 95\% CI 1.077, 1.386). Wasted children were 1.557 times more likely to be anemic compared to those of not wasted (AOR: 1.557, 95\% CI 1.315, 1.844) (Table 3).

\section{Discussion}

In this study, the prevalence of malnutrition and associated factors in Ethiopia was assessed. The prevalence of stunting, underweighting, and wasting were $38.3 \%$, $23.3 \%$, and $10.1 \%$ respectively. These prevalence were relatively lower than the previous study conducted in Ethiopia $[9,10]$ and in Tanzania [5], but it was higher than the study conducted in Nairobi, Kenya [11].
In this study, as the age of a child increase, the probability of a child to be stunted and underweighted will be increased. This finding was in line with the studies that conducted in Ethiopia, in which poor nutritional status of children was associated with the old age of children [12-14]. In all the three forms of malnutrition (stunting, underweight and wasting), the risk of malnutrition was less prevalent among females than males. This finding was consistent with previous findings [15-17]. This study revealed that the levels of malnutrition had a significant regional variation ranging from $14.6 \%$ in Addis Ababa to over $46.7 \%$ in Amhara regions of the country. This finding is similar with $[10,18]$.

Children whose mothers had primary and above educational level were significantly less likely to be stunted and underweighted as compared to children whose mothers had never attended formal education. This finding was consistent with the study conducted in Ethiopia [10] and Bangladesh [19] which showed that as mothers' educational level increase, the likelihood of the children to be stunted and underweighted will be decreased. Mothers with BMI less than 18.5 (underweight) were more likely to have stunted, underweighted and wasted children as compared to overweighted mothers. This finding is similar with other previously conducted studies $[10,19,20]$.

As of this study, children who were smaller at birth were more likely to be stunted and underweighted. This finding was supported by study conducted previously in SNNPR, Ethiopia. [13]. A similar study that conducted in Dale Woreda, Southern Ethiopia showed that the larger the family size, the poorer nutritional status of children would be resulted [17]. In the current study, anemia and malnutrition of children were highly associated with that anemic children were more likely to be malnutrition as compared to non-anemic [7].

\section{Conclusions}

The prevalence of stunting was still high in Ethiopia. The key determinants of malnutrition in Ethiopia were the child age, maternal education, region, household wealth status, religion, sex of child, number of children, a child size, water and toilet facility. The influence of

Table 3 Association of malnutrition with anemia among under-five children in Ethiopia

\begin{tabular}{llcccc}
\hline Variables & Category & Not anemic & Anemic (\%) & P-value & AOR (95\% Cl) \\
\hline Stunting & Not stunted & $2207(45.7)$ & $2619(54.3)$ & - & 1 \\
& Stunted & $1347(39.0)$ & $2106(61.0)$ & $<0.001$ & $1.222(1.101,1.356)$ \\
Underweight & Not underweight & $2798(45.4)$ & $3371(54.6)$ & - & 1 \\
& Underweight & $751(35.7)$ & $1354(64.3)$ & 0.002 & $1.222(1.077,1.386)$ \\
Wasting & Not wasted & $3295(44.1)$ & $4183(55.9)$ & - & 1 \\
& Wasted & $253(31.8)$ & $543(68.2)$ & $<0.001$ & $1.557(1.315,1.844)$ \\
\hline
\end{tabular}

AOR: adjusted odds ratio; $\mathrm{Cl}$ : confidence interval 
these factors should be considered to develop strategies for reducing malnutrition in Ethiopia. Finally, improving living standards of children is important to get a better health care, reduces child malnutrition, and child mortality.

\section{Limitations of the study}

This study was based on cross-sectional study design. Thus, the authors did not see the seasonal variation of malnutrition status and establish causal relationship. There were some missing values for some variables in the dataset. Therefore, the authors fail to consider some important factors which could affect the interpretation of the results.

\section{Additional files}

Additional file 1. Characteristics of the Study Participants (EDHS, 2016). Descriptive statistics of study variables.

Additional file 2. Results of multivariable logistic regression to identify the significant determinants related to wasting

\section{Abbreviations}

SPSS: Statistical Package for Social Science; COR: crude odds ratio; AOR: adjusted odds ratio; EDHS: Ethiopian Demographic and Health Survey; CSA: Central Statistical Agency; BMl: body mass index; WHO: World Health Organization; SNNPR: Southern Nations, Nationalities and People Region.

\section{Acknowledgements}

We would like to thank Statistics department staff of Bahir Dar University for their valuable suggestion and encourage. We also acknowledge Ethiopia Central Statistical Agency for giving us permission to use the data for our study.

\section{Authors' contributions}

AKT designed the current study, edited, analyzed the data and interpreted the results, and wrote the manuscript. AAW and GWB participated in the data analysis, manuscript writing, and acted as second reviewer. All authors read and approved the final manuscript.

\section{Funding}

Not applicable.

\section{Availability of data and materials}

The datasets used and analyzed during the current study are available from the corresponding author on reasonable request (in SPSS code).

\section{Ethics approval and consent to participate}

Since the data was obtained from Ethiopian Central Statistical Agency, the ethical clearance for the survey was approved by Ethical Review Board of Ethiopia Central Statistical Agency (CSA) and all participants who agreed to take part in the survey signed a consent form.

\section{Consent for publication}

Not applicable.

\section{Competing interests}

The authors declared that they have no competing interest.
Received: 11 February 2019 Accepted: 5 July 2019

Published online: 11 July 2019

\section{References}

1. WHO. Malnutrition - the global picture 2000. Geneva: WHO; 2000

2. WHO. Communicable disease and severe food shortage technical note. Geneva:WHO; 2010.

3. Black RE, Victora CG, Walker SP, Bhutta ZA. Maternal and child undernutrition and overweight in low-income and middle-income countries. Lancet. 2013;382(9890):427-51.

4. EDHS. Central Statistical Agency [Ethiopia] and ORC Macro. Ethiopia Demographic and Health Survey (EDHS); 2011.

5. Mgongo M, Chotta NAS, Hasham TH, Uriyo JG, Darmian DJ. Underweight, stunting and wasting among children in Kilimanjaro Region, Tanzania; a population-based cross-sectional study. Int J Environ Res Public Health. 2017:14(509):1-12.

6. Debela BL, Shively G, Holden S. Does Ethiopia's productive safety net program improve child nutrition? Food Secur. 2015;7(6):1273-89.

7. Teji K, O'Connor TP, Belachew T, O'Brien NM. Anemia and undernutrition among children aged 6-23 months in two agroecological zones of rural Ethiopia. Pediatric Health Med Ther. 2016;7:131-40.

8. EDHS. Central Statistical Agency [Ethiopia] and ORC Macro. Ethiopia Demographic and Health Survey (EDHS); 2016.

9. Asfaw M, Wondaferash M, Taha M, Dube L. Prevalence of undernutrition and associated factors among children aged between six to fifty nine months in Bule Hora district, South Ethiopia. BMC Public Health. 2015:15(41):1-9.

10. CSA. Key Findings on the 2014 urban employment unemployment survey: Addis Ababa, Addis Ababa, Ethiopia and Calverton, Maryland, USA; 2014

11. Chesire EJ, Oago ASS, Oteba LP, Echoka E. Determinants Of under nutrition among school age children in a Nairobi peri-urban slum. East Afr Med J. 2008;85(10):471-9.

12. Endris $\mathrm{N}$, Asefa $\mathrm{H}$, Dube L. Prevalence of malnutrition and associated factors among children in rural Ethiopia. Biomed Res Int. 2017;2017:6.

13. Ayalew $E$. The prevalence of stunting and associated factors among children age 6-59 months at Mizan-Aman Town, Bench Maji Zone, SNNPR Region, Ethiopia; 2015

14. Terefe D, Amare T, Gashaw AB, Molla MW. Stunting, wasting and associated factors among children aged 6-24 months in Dabat health and demographic surveillance system site: a community based cross sectional study in Ethiopia; 2017.

15. Demissie S, Worku A. Magnitude and factors associated with malnutrition in children 6-59 months of age in pastoral community of Dollo Ado district, Somali region, Ethiopia. Sci J Public Health. 2013;1(4):175-83.

16. Debeko DD, Goshu TA. Nutritional status of under-five children in Hawassa Zuria District, Southern Ethiopia. Am J Health Res. 2015:3(5):286-92

17. Wolde M, Berhan Y, Chala A. Determinants of underweight, stunting and wasting among schoolchildren. BMC Public Health. 2015;15(8):1-9.

18. Megabiaw B. Prevalence and determinants of chronic malnutrition among under-5 Children in Ethiopia. Int J Child Health Nutr. 2013:2:230-6.

19. Saddiqi MNA, Haque MN, Goni MA. Malnutrition of under-five children: evidence from Bangladesh. Asian J Med Sci. 2011;2011(2):113-9.

20. Yisak H, Gobena T, Mesfin F. Prevalence and risk factors for under nutrition among children under five at Haramaya district, Eastern Ethiopia. BMC Pediatr. 2015;15(212):1-7.

\section{Publisher's Note}

Springer Nature remains neutral with regard to jurisdictional claims in published maps and institutional affiliations. 